\title{
EFEMP1 binds the EGF receptor and activates MAPK and Akt pathways in pancreatic carcinoma cells
}

\author{
Peter Camaj, ${ }^{1, a}$, Hendrik Seeliger ${ }^{1, a}$, Ivan \\ Ischenko', Stefan Krebs², Helmut Blum², Enrico \\ N. De Toni ${ }^{3}$, Dagmar Faktorova ${ }^{4}$, Karl-Walter \\ Jauch $^{1}$ and Christiane J. Bruns ${ }^{1, *}$ \\ ${ }^{1}$ Department of Surgery, Munich University Medical \\ Center, Campus Großhadern, Marchioninistr. 15, \\ D-81377 Munich, Germany \\ ${ }^{2}$ Laboratory for Functional Genome Analysis (LAFUGA), \\ Gene Center, Ludwig Maximilians University, D-81377 \\ Munich, Germany \\ ${ }^{3}$ Department of Gastroenterology, Munich University \\ Medical Center, Campus Großhadern, Marchioninistr. \\ 15, D-81377 Munich, Germany \\ ${ }^{4}$ Department of Measurement and Applied Electrical \\ Engineering, University of Zilina, SK-01026 Zilina, \\ Slovak Republic \\ ${ }^{*}$ Corresponding author \\ e-mail: Christiane.Bruns@med.uni-muenchen.de
}

\begin{abstract}
The EGF-related protein EFEMP1 (EGF-containing fibulin-like extracellular matrix protein 1) has been shown to promote tumor growth in human adenocarcinoma. To understand the mechanism of this action, the signal transduction activated upon treatment with this protein has been investigated. We show that EFEMP1 binds EGF receptor (EGFR) in a competitive manner relative to epidermal growth factor (EGF), implicating that EFEMP1 and EGF share the same or adjacent binding sites on the EGFR. Treatment of pancreatic carcinoma cells with purified EFEMP1 activates autophosphorylation of EGFR at the positions Tyr-992 and Tyr-1068, but not at the position Tyr-1048. This signal is further transduced to phosphorylation of Akt at position Thr-308 and p44/p42 MAPK (mitogen-activated protein kinase) at positions Thr-202 and Tyr-204. These downstream phosphorylation events can be inhibited by treatment with the EGFR kinase inhibitor PD 153035. The observed signal transduction upon treatment with EFEMP1 can contribute to the enhancement of tumor growth shown in pancreatic carcinoma cells overexpressing EFEMP1.
\end{abstract}

Keywords: downstream signaling; EFEMP1; EGF receptor (EGFR); epidermal growth factor (EGF); phosphorylation.

a These authors contributed equally to this work.

\section{Introduction}

Fibulins are a family of five extracellular matrix proteins characterized by tandem arrays of epidermal growth factor (EGF)-like domains and a C-terminal fibulin-type module. They are widely distributed and often associated with vasculature and elastic tissues (Kobayashi et al., 2007). EFEMP1 (EGF-containing fibulin-like extracellular matrix protein 1) also known as fibulin-3 plays an important role in many physiological processes including pathogenesis of degenerative eye diseases. Age-related macular degeneration (AMD) is the most common cause of vision loss in developed countries. EFEMP1 R345W mutant mice develop deposits of material between Bruch's membrane and the retinal pigment epithelium, which resemble basal deposits in patients with AMD (Fu et al., 2007). The same mutation is responsible for the eye disease Malattia Leventinese, indicating that EFEMP1 might be important in drusen formation. Wild type EFEMP1 is a secreted protein, whereas mutant EFEMP1 R345W is misfolded, secreted inefficiently, and retained within cells (Marmorstein et al., 2002). Data obtained on EFEMP1/-- mice have confirmed the hypothesis about the role of misfolding in manifestation of symptoms of this disease. No apparent macular degeneration associated defects were found in these mice, suggesting that loss of fibulin- 3 function is not the mechanism by which the mutation in EFEMP1 causes macular degeneration (McLaughlin et al., 2007). Nevertheless, EFEMP1 knockin mice carrying disease-associated mutation in the murine EFEMP1 gene reconstitute the most important histopathologic symptoms of both Malattia Leventinese and AMD (Marmorstein et al., 2007).

EFEMP1 also participates in other processes such as regulation of body weight or behavioral control. $\mathrm{A} \mathrm{C/T}$ polymorphism of the EFEMP1 gene in the position 56022960 of chromosome 2 is one of the identified variants influencing adult human height (Weedon et al., 2008). Transcriptional analysis of 11 post-mortem brain tissues of suicide victims compared with 10 non-psychiatric controls revealed that changes on EFEMP1 expression could be implicated in the etiology of human suicidal behavior (Thalmeier et al., 2008). These data suggest that EFEMP1 is subject to different cellular regulation mechanisms.

In the current study, we demonstrated an interaction of EFEMP1 of wild type with EGF receptor (EGFR) followed by the activation of downstream signaling in a human pancreatic adenocarcinoma cell line. EFEMP1 is for the first time shown as a functional ligand of the EGFR activating Akt- and mitogen-activated proteinkinase (MAPK)-signal transduction. 


\section{Results}

\section{Expression of EFEMP1 and other relevant genes in pancreatic carcinoma cells}

Our previous Affymetrix analysis of transcriptome of L3.6pl cells vs. FG cells has revealed that EFEMP1 belongs to the group of the genes being upregulated in the aggressive, highly metastatic and angiogenic pancreatic carcinoma cell line L3.6pl. Our quantitative real-time RT-PCR data have fully confirmed these data (Seeliger et al., 2009). The transcription results by themselves, however, do not reveal biological relevance. Therefore, we investigated expression of this gene on the protein level in both previously studied cell lines via Western blotting using anti-EFEMP1 specific antibody. Our results show that the expression of EFEMP1 is upregulated on the protein level as well in the cell line L3.6pl (Figure 1). We have shown that elevated expression of EFEMP1 is responsible for at least the part of differential phenotype of L3.6pl vs. FG cells (Seeliger et al., 2009).

RT-PCR data revealed no significant differential gene expression of EGFR in both investigated cell lines determined on both RNA and protein levels (Figure 2). Our FACE $^{\text {TM }}$ (Fast Activated Cell-based ELISA) results confirmed the results on total EGFR content obtained by Western blotting and also demonstrated significant differences in phosphorylation of EGFR at the position of Tyr-992 in both investigated cell lines.

EGF content and production was also investigated. The growth medium containing 10\% fetal calf serum (FCS, batch: S0115, Biochrom, Berlin, Germany) does not contain EGF in an amount detectable by ELISA. RTPCR data revealed weak transcription of the EGF gene in both investigated cell lines. The results of EGF ELISA with conditioned media reveal that both investigated cell lines produce only $3 \mathrm{pg}$ of EGF per $\mathrm{ml}$ of medium and per million of cultivated cells during $48 \mathrm{~h}$. These data show that the EFEMP1 protein, being described for the first time in the context of cancer, might play an important role in carcinogenesis. Therefore, we investi-
$\mathrm{F}$

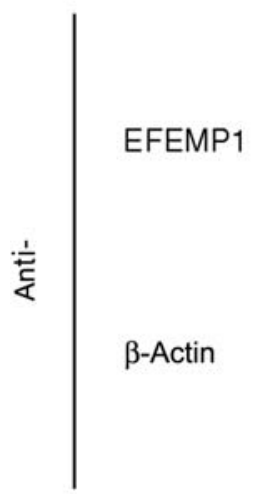

$\mathrm{L}$
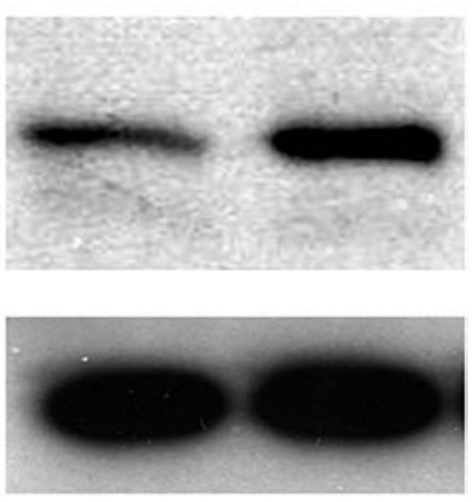

Figure 1 Expression of EFEMP1 in FG and L3.6pl cells. Results of Western blotting performed using an anti-EFEMP1 antibody and a $\beta$-actin antibody as a loading control. ' $F$ ' denotes cell extract prepared from $F G$ cells and ' $L$ ' denotes the cell extract prepared from L3.6pl cells. gated the function of this protein in terms of signal transduction.

\section{Sequence of EFEMP1 L3.6pl-cDNA}

Our results have shown that the cells overexpressing EFEMP1 exhibit elevated proangiogenic properties (Seeliger et al., 2009). It has been previously described that the mutation R345W causes poor secretion of EFEMP1 protein following upregulation of VEGF expression (Roybal et al., 2005). Therefore, cDNA has been amplified by PCR and sequenced. Our results show that both cell lines, L3.6pl and FG, express wild type EFEMP1 gene coding for arginine in the position 345.

\section{Interaction of EFEMP1 with EGFR}

The multiple sequence alignment revealed significant similarity of amino acid sequences between 1207 amino acid long form of EGF as the primary ligand of EGFR and EFEMP1 (Figure 3). Hot spot of similarity is concentrated in the amino acid sequence position 53 creating the structure of soluble EGF interacting with its receptor. Therefore, we addressed the issue of a possible interaction between EFEMP1 (molecular mass of $54 \mathrm{kDa}$ ) and EGFR. Following immunoprecipitation using anti-EGFR antibody, the band corresponding to the protein with an apparent molecular mass of approximately $54 \mathrm{kDa}$ was detected in the case of both extracts prepared from FG or L3.6pl cells, respectively. The band was specifically identified by anti-EFEMP1 antibody and was dependent on the presence of anti-EGFR antibody during immunoprecipitation (see control without anti-EGFR antibody on Figure 4). The presented data show that EFEMP1 has the ability to interact with EGFR.

\section{Competition between EFEMP1 and EGF on binding to its receptor}

To investigate whether EFEMP1 competes with EGF for binding to its receptor, the described immunoprecipitation experiment was repeated after treatment of the L3.6pl cells with EGF or with bovine serum albumin (BSA) as an unspecific control, respectively. The amount of cell extract used for immunoprecipitation was normalized to the same protein concentration. Additionally, the amount of immunoprecipitated EGFR was tested by Western blotting using anti-EGFR antibody. Our data (Figure 5) showed that the amount of immunoprecipitated EFEMP1 after treatment with anti-EGFR antibody is significantly reduced in competition with EGF, whereas the amount of immunoprecipitated EGFR is comparable.

\section{Phosphorylation of EGFR induced by EFEMP1}

Interaction between EGF and its receptor leads to an activation of receptor self-phosphorylation (Koland et al., 1990). Therefore, we investigated phosphorylation of EGFR in the pancreatic cell line L3.6pl treated with purified EFEMP1 protein. Western blotting using the extract of cells treated with $50 \mathrm{ng} / \mathrm{ml}$ EFEMP1 showed that this treatment activates phosphorylation of EGFR at the posi- 
A

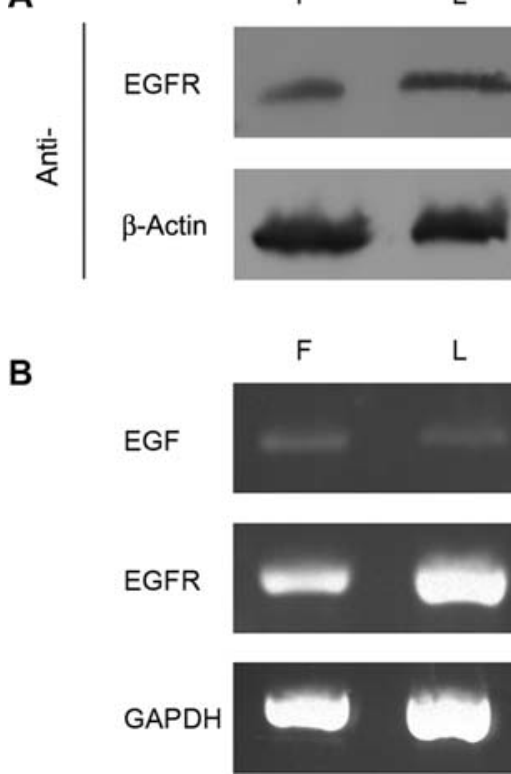

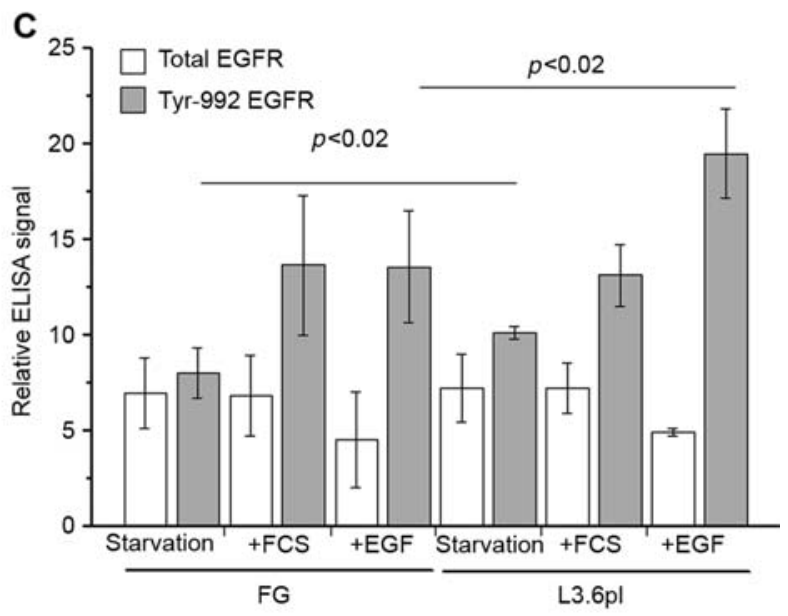

Figure 2 Expression of EGFR (epidermal growth factor receptor) in FG or L3.6pl cells respectively.

(A) Western blotting. Proteins from cell extracts prepared from both cell lines were subjected to Western blotting and detected using the anti-total-EGFR antibody. Loading was controlled using anti- $\beta$-actin antibody. ' $F$ ' denotes FG cells and ' $L$ ' denotes L3.6pl cell line. (B) Transcription of relevant gene in FG or L3.6pl cells, respectively, analyzed by RT-PCR. Total RNA prepared from both untreated cell lines was subjected to single step RT-PCR. Its products were separated in the agarose gel. 'F' denotes FG cells and 'L' denotes L3.6pl cell line. Used primer sets are indicated. (C) Results of FACE ${ }^{\mathrm{TM}}$ in cell Western blot analysis for Y992 EGFR. The same amount of FG and L3.6pl cells were serum-starved for $24 \mathrm{~h}$ and then activated with FCS or EGF, or were left untreated. Colorimetric signal proportional to concentration of EGFR or to its activation on Tyr-992, respectively, was recorded and normalized to the signal obtained after crystal violet staining proportional to the cell number. Relative values are depicted; treatments and used cell lines are indicated.

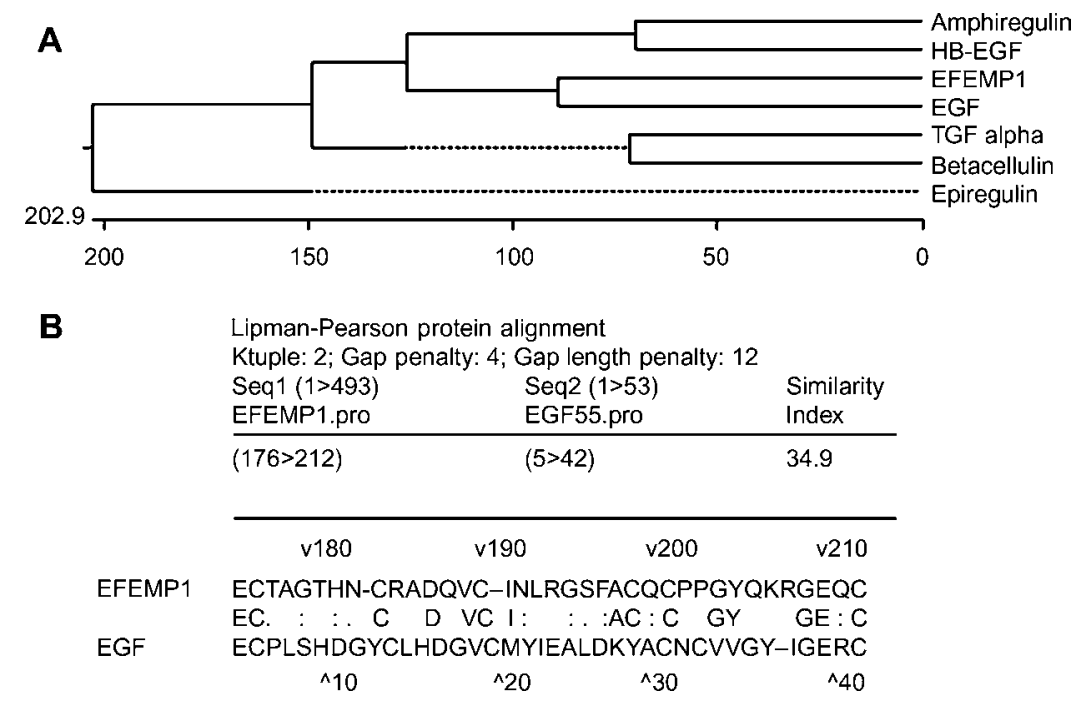

Figure 3 Results of the multiple sequence alignment.

(A) Shown in the form of a phylogenetic tree. The sequences of the EGF receptor ligands were compared using DNA* MegAlign software. The similarity between the sequences of EFEMP1 and EGF is shown. (B) Lipman Pearson protein sequence alignment EFEMP1 and EGF. Analysis was performed using DNA* MegAlign software. Consensus sequence and amino acid similarity between both sequences are indicated.

tions of Tyr-992 and Tyr-1068. There was no significant difference between phosphorylation of Tyr-1048 in EGFR in the samples treated or untreated with EFEMP1 (Figure 6). Probing of the membrane with antibody identifying the total amount of EGFR showed equal gel-loading.

\section{Effect of EFEMP1 treatment on Akt and MAPK signaling}

Because our data showed that EFEMP1 has the ability to bind EGFR and that this binding triggers its phospho- 


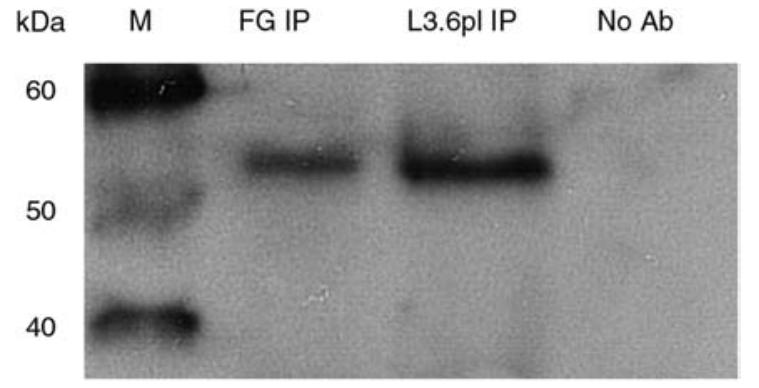

Figure 4 Co-immunoprecipitation of EFEMP1 with the EGF receptor.

Anti-EGFR antibody was used for immunoprecipitation of the same protein amount of the cell extract made on FG or L3.6pl cells, respectively. The immunoprecipitate was probed with antiEFEMP1 antibody using immunoblotting. The positive band corresponds to the protein being identified by the antibody, and its electrophoretic mobility corresponds to a molecular mass of $54 \mathrm{kDa}$ for EFEMP1. The molecular masses according to Magic XP Western marker are indicated. FG IP denotes the proteins immunoprecipitated from FG cell extract and L3.6pl IP denotes the proteins immunoprecipitated from L3.6pl cell extract. As a negative control, a sample in the absence of the anti-EGFR antibody (noAb) was used.

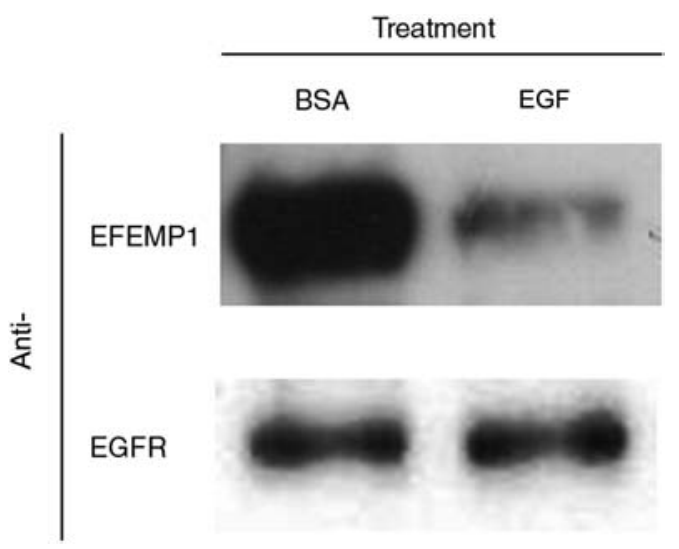

Figure 5 Competition between EGF and EFEMP1 on binding to EGFR.

Immunoprecipitation using anti-EGFR antibody was repeated after treatment with $50 \mathrm{ng} / \mathrm{ml}$ EGF or with irrelevant arbitrary protein, BSA. Treatment with EGF diminishes the yield of the immunoprecipitated EFEMP1 probably owing to competition between EGF and EFEMP1 binding to EGFR. The membrane was reprobed with the antibody identifying the total immunoprecipitated EGFR as a loading control. BSA denotes the cell extract prepared on cells treated with BSA and EGF denotes the cell extract prepared on cells treated with EGF.

rylation, we investigated activation of downstream targets: Akt and Erk2. The extract prepared from L3.6pl cells treated with $50 \mathrm{ng} / \mathrm{ml}$ EFEMP1 or with the same concentration of BSA were normalized to the same protein concentrations and were subjected to Western blotting with phosphorylation-sensitive antibodies against Akt and Erk2. Our data show that treatment of L3.6pl cells with EFEMP1 activates the MAPK pathway, as demonstrated by phosphorylation of Erk2 proteins p42 and p44 in positions Tyr-202 and Tyr-204, respectively (Figure 7). Similarly, EFEMP1 treatment activates phosphorylation of Akt in position Thr-308 which has been

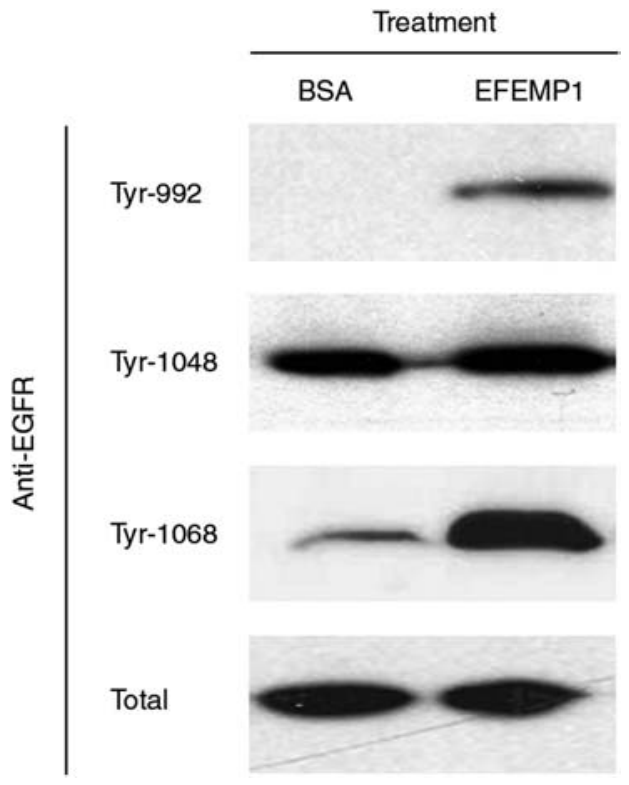

Figure 6 Autophosphorylation of EGFR on treatment with EFEMP1.

Biological relevance of EFEMP1 binding on the EGFR was demonstrated by detection of autophosphorylation of EGFR on treatment with $50 \mathrm{ng} / \mathrm{ml}$ of purified EFEMP1 or with the same amount of arbitrary protein BSA used as a negative control. Phosphorylation of EGFR on the sites Tyr-992, Tyr-1048, and Tyr-1068 on treatment with EFEMP1 was monitored by immunoblotting using appropriate anti-phospho-EGFR antibody. The membrane was also probed with the antibody identifying total EGFR as a loading control.

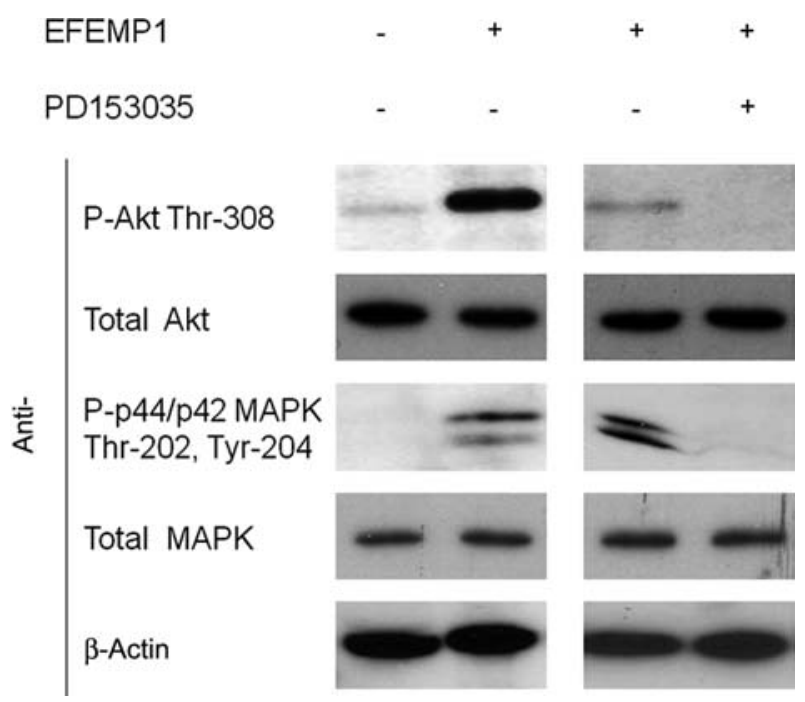

Figure 7 Effect of EFEMP1 treatment on downstream MAPK and Akt signaling.

Activation of MAPK- and Akt-signal transduction on treatment of L3.6pl cells with EFEMP1 was demonstrated by immunoblotting with anti-phospho-p44/p42 MAPK (Erk) Thr-202, Tyr-204, and phospho-Akt Thr-308 antibodies. Treatment of L3.6pl cells leads to activation of p44/p42 MAPK in positions Thr-202 and Tyr-204, and to activation of Akt in position Thr-308 as well. This effect can be reversed on treatment with EGFR inhibitor PD135035. The membrane was probed with anti- $\beta$-actin, total Akt, and total MAPK antibodies for a loading control. Treatments and the antibodies are indicated. 
demonstrated via immunoblotting using appropriate antiphospho-Akt antibody.

\section{Treatment with EGFR inhibitor PD153035}

If phosphorylation of downstream proteins such as Akt and Erk2 is caused by the activation of EGFR signaling upon treatment with EFEMP1, inhibition of EGFR activity should remove this effect. To test this hypothesis, L3.6pl cells were treated with $50 \mathrm{ng} / \mathrm{ml}$ EFEMP1 in combination with the EGFR inhibitor PD153035 $(2 \mu \mathrm{mol} / \mathrm{ml})$ or with $50 \mathrm{ng} / \mathrm{ml}$ EFEMP1 alone. Cell extracts were normalized to the same protein concentration. Data obtained after Western blotting using anti-phospho-Akt (Thr-308) antibody and anti-phospho-p44/42 MAPK (Thr-202/Tyr-204) show that the inhibition of EGFR via PD153035 abrogates activation of Akt and Erk2 after EFEMP1 treatment. These data suggest that the activation of Akt- and MAPK-signal transduction pathways is mediated through the EGFR-signaling pathway.

\section{Discussion}

\section{Overexpression of EFEMP1 in highly metastatic pancreatic carcinoma cells}

Our data show that EFEMP1 is overexpressed in highly metastatic and proangiogenic cell line L3.6pl (Bruns et al., 1999) in comparison with its less metastatic maternal cell line FG. Overexpression has been observed on both the transcriptional and translational levels (Seeliger et al., 2009; Figure 1). Interestingly, our Affymetrix analysis, previously published data (Nakamura et al., 2007; Seeliger et al., 2009) as well as our RT-PCR and Western blotting data do not exhibit significant differences in gene expression on the transcription and translation levels (Figure 1). At the same time, differential activation of EGFR via phosphorylation of Tyr-992 was demonstrated (Figure 2C). Our previously published data (Bruns et al., 2000) demonstrated that the activation of EGFR plays an important role in growth of tumors initiated by implantation of L3.6pl cells into nude mice. Because the growth medium does not contain any EGF and cells produce very little of this growth factor, EGFR activation observed in a cell culture has to be dependent on an activation of the receptor via alternative ligands or via crosstalk with other cell signaling. We have previously demonstrated that EFEMP1 is upregulated in L3.6pl cells (Seeliger et al., 2009). Therefore, we assume that EFEMP1 contributes at least partially to the establishment of the aggressive phenotype of these cells. Presented data lead to the hypothesis that the effect of EFEMP1 on tumorigenesis could be mediated via modulation of EGFR signaling.

\section{Binding of EFEMP1 to EGFR}

Based on the primary structure of EFEMP1, the protein belongs to the family of the proteins bearing the EGF domain. Therefore, we compared sequences of all known EGFR-binding proteins with the EFEMP1 sequence using the ClustalV algorithm. Of all known EGFR-binding pro- teins EFEMP1 has the highest homology to EGF (Figure $3 A)$. Sequence alignment demonstrated that homology is focused on the hot spot (Figure 3B), which is important for binding of EGF to its receptor classified by X-ray crystallographic data (Ogiso et al., 2002). Replacements of Ile23 with Ala, Val, Leu, Phe, Trp, etc., have shown that Ile23 of EGF is requisite for tight binding with the receptor (Koide et al., 1992). The EGF lle23-binding site, formed by the side chains of Leu14, Tyr45 and Leu69 of EGFR, has a shape roughly complementary to that of the lle side chain. The residues 31-33 of EGF form a short parallel $\beta$-sheet important for interaction with EGFR (Ogiso et al., 2002). The residues 31 and 33 of EGF are identical with their corresponding amino acids 199 and 201 of EFEMP1 (Figure 3B). Asn32 of EGF is in EFEMP1 substituted with similar amino acid Gln200. In addition, the Gln16 side chain of EGFR creates hydrogen bonds with the Asn32 side chain of EGF (Ogiso et al., 2002). In this context, the replacement of Asn32 by Asp significantly reduced the receptor binding activity of EGF, but its replacement by His, which can mimic Asn with regard to the hydrogen donor activity, did not (Koide et al., 1992). Features of GIn are more similar to Asn than features of His. Obviously, EFEMP1 contains appropriate amino acids in positions important for binding with EGFR.

Based on these facts we assumed that EFEMP1 could belong to the EGFR ligand group. This hypothesis was confirmed by immunoprecipitation experiments. Only in the presence of anti-EGFR antibody, a protein with an apparent molecular mass of $55 \mathrm{kDa}$ and being specifically identified by anti-EFEMP1 antibody (Figure 4) can be selectively co-immunoprecipitated. The molecular mass of EFEMP1 according to its amino acid sequence is $54640 \mathrm{Da}$.

Because on the one hand EFEMP1 binds EGFR, and on the other hand it is homologous to EGF at its EGFRbinding interface, we assumed that both ligands might bind to the same region of the receptor. Then EFEMP1 and EGF would compete for binding to the same receptor. Therefore, we repeated the same immunoprecipitation experiment with the cells pretreated with the potential competitor, EGF. In fact, yield of EFEMP1 immunoprecipitated in the presence of added EGF was diminished in comparison with the yield of EFEMP1 immunoprecipitated using the control cell extract prepared on the cells treated with an arbitrary protein, BSA. The results show that EFEMP1 and EGF share the same EGFR region for their binding.

\section{Activation of autophosphorylation on treatment with EFEMP1}

It has been previously shown that interaction of EGFR with its ligands leads to the activation of autophosphorylation of the receptor at specific amino acids (Koland et al., 1990). This action is accompanied by the activation of downstream signaling and has been observed not only on binding of EGF but also on binding of other EGF ligands such as HB-EGF (Yin and Yu, 2009). Therefore, the phosphorylation pattern of EGFR on treatment with the purified EFEMP1 was investigated. Basal level of phosphorylation of EGFR in absence of EFEMP1 was determined and the treatment with BSA was used as a 
control. Immunodetection using site-specific anti-phospho-antibodies revealed that EGFR is specifically phosphorylated in the positions Tyr-992 and Tyr-1068 on treatment with purified EFEMP1. The treatment of L3.6pl cells with EFEMP1 does not affect autophosphorylation of EFGR in the position Tyr-1048. This means that EFEMP1 binding does not activate the interaction with Grb2 and therefore inhibition of the Ras-signaling and proteasomal degradation of EFGR are not activated (Rojas et al., 1996). Activation of EGFR autophosphorylation at the positions Tyr-992 and Tyr-1068 shows that EFEMP1-based signal could be further transduced toward MAPK and Akt activation (Zhang et al., 2002).

\section{Activation of the downstream signaling}

To investigate relevance of EFEMP1-binding on EFGR for downstream signaling, we treated L3.6pl cells with purified EFEMP1 or with BSA as unspecific control, respectively. Both cell extracts were subjected to Western blotting using antibodies specifically identifying activated forms of downstream EGFR-signaling targets deduced according to identified pattern of autophosphorylation induced by EFEMP1. Results suggested that signals caused by EFEMP1-binding to EGFR are transduced further to phosphorylation of downstream effectors. It has been previously shown that stimulation of the pancreas carcinoma cells with EGF or FCS leads to the activation of ERK1 (extracellular regulated proteinkinase 1) and p42 MAPK. MEK-1 inhibitor PD98059 abolished EGF- and FCS-induced MAPK activation (Giehl et al., 2000). EGF treatment of human Panc-48 adenocarcinoma cells activated Akt and Erk pathways. At the same time, the blockade of the phosphatidylinositol-3 kinase/ Akt and MAPK/Erk abrogated the EGF-induced downstream signaling (Parikh et al., 2003; Yip et al., 2008). Therefore, EFEMP1 activation of the downstream MAPKand Akt- signaling in the presence or absence of inhibitor of the EGFR autophosphorylation, PD153035 (Fry et al., 1994), was investigated. The results show that both Aktand MAPK-signal transduction pathways can be activated in the form of Akt phosphorylation in the position Thr-308 and p42/p44 MAPK phosphorylation in the positions Thr-202 and Tyr-204, on treatment of the L3.6pl cells with purified EFEMP1 (Figure 7). This activation could be abrogated via the treatment with inhibitor of the EGFR autophosphorylation. These data indicate that the kinase activity of EGFR is essential for the transduction of the EFEMP1 signal downstream toward activation of Akt and MAPK pathways.

\section{Biological relevance of binding EFEMP1 to EGFR for pancreas carcinoma cells}

Our study shows specific activation of Akt and MAPK pathways under treatment with the protein EFEMP1 upregulated in in vivo selected highly metastatic pancreas carcinoma cell lines relative to their low metastatic unselected counterpart. Using stable EFEMP1 transfectants, we have shown that overexpression of this gene causes enhanced tumor growth, metastasis and angiogenesis in vivo (Seeliger et al., 2009). These data demonstrate that upregulation of EFEMP1 in L3.6pl cells is at least co-responsible for the enhancement of angiogenesis and metastasis rates occurring during the in vivo selection leading to the transformation of less aggressive cells FG to very highly aggressive cells L3.6pl. These effects could be caused by an additional activation of EGFR as a result of EFEMP1 binding followed by downstream Akt and MAPK activation. In some situations both EGFR and EFEMP1, as one of its ligands, are regulated simultaneously. The expression levels of EGFR and EFEMP1 of human coronary artery endothelial cells are downregulated under the exposition to laminar shear stress (Chu and Peters, 2008).

Akt- and MAPK-signal transduction is an important mediator of several cellular fates including growth, proliferation and survival in several tissues including pancreatic tumors (Takeda et al., 2004; Schonleben et al., 2007). Activation of the tumor growth as a result of EGFsignaling transduced via MAPK or Akt activation is a more general scenario present in several cell types (Walker et al., 1998; Schmitz et al., 2008). Net cell growth is a result of a shift in the equilibrium between cell proliferation and cell death. EGF-induced MAPK activation in the $\mathrm{BaF} / 3$ cells inhibits apoptosis but does not influence proliferation (Walker et al., 1998).

PI3K (phosphatidyl-inositol-3 kinase) and Akt mediate survival and proliferative signals, allowing the cells to escape apoptosis in various human cancers. PI3K inhibition significantly enhances the antiproliferative and proapoptotic effects of TNF $\alpha$ in both pancreas carcinoma cell lines Panc-1 and MiaPaCa-2 (Shah et al., 2001; Yao et al., 2002). In patients, $63-70 \%$ of the pancreas tumors overexpress phosphorylated Akt (Semba et al., 2003). Pancreatic cancer cells treated with PI3K inhibitor, LY294002, underwent G1 arrest, which was associated with the dephosphorylation of the ppRB protein, a decrease in the protein expression of cyclin $D$ and cyclin $\mathrm{E}$, and their activating partners $\mathrm{Cdk} 2,4$, and 6 with simultaneous accumulation of P27/Kip1. This accumulation by Akt inactivation could induce the cell cycle arrest in the G1 phase and suggest that the PI3K-Akt pathway plays an important role in cell proliferation in human pancreatic ductal carcinoma cells (Takeda et al., 2004).

Mitogen-activated protein kinases are known to regulate apoptosis in various cancers. SB202190, an inhibitor of p38, prevented 15d-PGJ2-induced activation of caspase-8, -9 , and -3 and significantly decreased apoptosis. PD98059, an inhibitor of MEK, significantly increased sensitivity of the pancreatic cancer cells to apoptosis (Hashimoto et al., 2004).

The role of EGF-signaling in chemoresistance has been previously shown in ovarian cancer cells. The EGFR ligand HB-EGF has been found to be upregulated in cancer cells and has been identified as an agent responsible for invasion, angiogenesis, and chemoresistance (Miyamoto et al., 2007). The cisplatin-resistant MCF-7 breast cancer cells are characterized by increased EGFR phosphorylation, high levels of Akt activity, and Erk1 phosphorylation (Eckstein et al., 2008). These data are in good agreement with our observation that FG cells stable transfected with a vector bearing the EFEMP1 gene exhibit enhanced chemoresistance, proliferation, and inhibited apoptosis (Seeliger et al., 2009). We speculate 
that EFEMP1-induced activation of EGFR-signaling activating Akt and MAPK pathways could be responsible for features of the EFEMP1 transfectants.

The issue of the effect of EFEMP1 overexpression on angiogenesis is controversial. Angiogenesis is one step of the pathogenic process of eye diseases caused by mutation in the EFEMP1 gene. Accumulation of misfolded mutant R345W EFEMP1 protein within the endoplasmic reticulum (ER) causes an activation of unfolded protein response signaling and expression of ER stressresponsive genes, including VEGF (Roybal et al., 2005). Our previous data show that the cells overexpressing EFEMP1 exhibit an enhanced angiogenetic potential in comparison with the control cell line (Seeliger et al., 2009). By contrast, it has been shown that constitutive expression of EFEMP1 in the endothelial cells leads to an inhibition of their invasion, proliferation, and sprouting (Albig et al., 2006). Despite that we have not observed any significant effect on the proliferation and apoptosis on treatment of HUVECs (human umbilical vein endothelial cells) with purified EFEMP1, our data are not in contradiction with this study. We have found that VEGF secretion is enhanced in FG cells overexpressing EFEMP1 in comparison with the control cell line. This implicates that not EFEMP1 directly but EFEMP1induced increased VEGF production is responsible for proangiogenic phenotype of EFEMP1 transfectants. Thus, our data presented in this study is in good agreement with the observations described above. It has been shown in L3.6pl cells that both the PI3K/Akt and p38 MAPK pathways are activated in a Src family kinasedependent manner on EGFR activation and are important for EGF-mediated VEGF production in pancreatic cancer cells (Summy et al., 2005). Therefore, we assume that the described mechanism could explain the role of EFEMP1induced EGFR activation in enhancement of angiogenesis via increased VEGF secretion.

The data presented in this study show that EFEMP1 protein overexpressed in highly metastatic pancreas carcinoma cell line L3.6pl binds to EGFR. EFEMP1 competes with EGF for receptor binding. EFEMP1 binds to EGFR at the EGF binding site or in the close neighborhood. This binding causes autophosphorylation of the receptor in the positions Tyr-992 and Tyr-1068, but not in the position Tyr-1068. Phosphorylation of EGFR leads to activation of downstream signaling via Akt and MAPK. This activation could be causative for the phenotype observed in EFEMP1 overexpressing cells: enhancement of proliferation, chemoresistance, and angiogenesis as well as inhibition of apoptosis.

\section{Materials and methods}

\section{Pancreatic cancer cell lines}

The pancreatic cancer cell line FG is a variant of Colo375, a human pancreatic adenocarcinoma cell line originally derived from a celiac lymph node metastasis. L3.6pl is a highly metastatic and angiogenic variant of FG selected by repeated injection of metastatic cells into the spleen and pancreas of mice. Both cell lines were maintained in Dulbecco's modified Eagle
Medium supplemented and under culture conditions as described previously (Bruns et al., 1999).

\section{Determination of concentration of EGF}

Concentration of EGF in the original growth media or in the media conditioned by both cell lines, respectively, was determined using a Human EGF Quantikine ELISA Kit (R\&D Systems, Minneapolis, USA) according to the manufacturer's instruction manual. The medium was conditioned by the same amounts of FG or L3.6pl cells, respectively, for $48 \mathrm{~h}$ under the conditions of the cell cultivation. Cell number was determined and the results were calculated in $\mathrm{pg} / \mathrm{ml} / 10^{6}$ cells.

\section{Sequencing of EFEMP1-cDNA}

To test whether the gene EFEMP1 is expressed in the cell lines FG and L3.6pl in a wild type form or in a proangiogenic mutant form (e.g., as mutant R345W), total RNA from both unstimulated cell lines was isolated using a RNeasy mini kit (Qiagen, Hilden, Germany). Total RNA was subjected to reverse transcription followed by PCR using the SuperScriptlll One-Step RT-PCR system with Platinum Taq High Fidelity (Invitrogen, Karlsruhe, Germany) using gene-specific primers:

\section{EFEMP1forw: 5'-GAG GGG AGC AGT GCG TAG ACA;} EFEMP1rev: 5'-TCG GCA CAT GGC ATT TGA GAC.

The expected $823 \mathrm{bp}$ long PCR product was detected by electrophoresis in $1 \%$ agarose gel with ethidium bromide staining. The PCR product was purified using a QIAquick PCR Purification Kit (Qiagen). Purified PCR product was sequenced by Sequencing Service LaFuGa (Munich, Germany). Sequencing data were edited and the sequence was compared with the published EFEMP1 sequence using DNA* SeqMan and MegAlign software (DNASTAR, Madison, USA).

\section{Quantitative RT-PCR}

To compare transcription of EFEMP1 in FG vs. L3.6pl cells, quantitative real-time PCR and semi-quantitative RT-PCR was applied to RNA extracted from both cell cultures using a RNeasy kit (Qiagen). RNA integrity was verified by agarose-formaldehyde electrophoresis detection of $18 \mathrm{~S}$ and $28 \mathrm{~S}$ rRNA, and isolated RNA was quantified by spectrophotometry using the GeneQuant Pro RNA/DNA calculator (Pharmacia, Freiburg, Germany). Quantitative RT-PCR for EFEMP1 was conducted using a SuperScriptIII Platinum SYBR green one-step qRT-PCR kit (Invitrogen) with the EFEMP1 gene-specific primers EFEMP1up and EFEMP1low (see above) according to the manufacturer's instructions on a LightCycler system (Roche Diagnostics, Mannheim, Germany). Typically, appropriate results were obtained after 45 cycles. PCR efficiency was assessed using the plasmid standards and quantified relative to the housekeeping gene transcript GAPDH. Sequences of the specific primers are as follows:

GAPDH forw: 5'-GAG TCA ACG GAT TTG GTC GTA TTG GGC G-3';

GAPDH rev: 5'-GAC GCC TGC TTC ACC ACC TTC TTG ATG TC-3';

EGF forw: 5'-TGA TTT GCC CTG ACT CTA CTC CAC CC-3'; EGF rev: 5'-GGC CTG CGA CTC CTC ACA TCT CTG-3'; EGFR forw: 5'-CGC CCA GAC CGG ACG ACA GG-3'; EGFR rev: 5'-CAC GGC GCC ATG CAG GAT TTC-3'.

To test the size of the PCR product, semi-quantitative RT-PCR was performed using the SuperScriptIII One-Step RT-PCR System with Platinum Taq High Fidelity (Invitrogen) using the same 
template and the same primer sets as in the case of real-time RT-PCR.

\section{Western blotting}

Proteins from cell lysates or from immunoprecipitation were separated in SDS-PAGE gel with an appropriate concentration of acrylamide/Bis to ensure optimal separation. Proteins were transferred onto PVDF (polyvinylidene difluoride) membrane Hybond P (Amersham/GE Healthcare, Uppsala, Sweden) via semi-dry blotting. Efficient blotting was demonstrated by transfer of the PeqGOLD prestained protein marker IV (PeqLab, Erlangen, Germany). The membrane was then incubated for $1 \mathrm{~h}$ at room temperature with Roti-Immunoblock buffer (Carl Roth, Karlsruhe, Germany). After washing with TTBS (TBS+ $0.5 \%$ Tween 20) buffer, the membrane was incubated with the first antibody diluted in TTBS to the working concentration and was incubated according to the manufacturer's data. After washing, the membrane was incubated with the HRP-labeled second antibody (Dako, Glostrup, Denmark) diluted in blocking buffer to the working concentration and incubated according to the manufacturer's data. After extensive washing, the bands were visualized using ECL Plus Western blotting reagents and Hyperfilm ECL (both from Amersham/GE Healthcare).

\section{Co-immunoprecipitation of EFEMP1 and EGFR}

Cell lysates were prepared from both cell lines L3.6pl and FG using Complete Lysis-M buffer (Roche). The amount of lysate was normalized to protein concentration assayed by a BSA Protein Assay Kit (Pierce, Rockford, IL, USA). To deplete the lysate on the proteins unspecifically bound to the agarose beads, $200 \mu \mathrm{l}$ of the lysate was incubated with protein G agarose beads (Sigma-Aldrich, Munich, Germany; $20 \mu \mathrm{l}$ of $50 \%$ bead slurry) with gentle rocking for $1 \mathrm{~h}$ at $4^{\circ} \mathrm{C}$ with no antibody present. Preadsorbed lysates were incubated with gentle rocking overnight at $4^{\circ} \mathrm{C}$. Then, $20 \mu \mathrm{l}$ of $50 \%$ bead slurry of protein $\mathrm{G}$ agarose and $10 \mu \mathrm{g}$ anti-EGFR antibody was added. The samples were incubated with gentle rocking for $3 \mathrm{~h}$ at $4^{\circ} \mathrm{C}$ and washed 5 times with $500 \mu \mathrm{l}$ of the cell lysis buffer. The pellet was then resuspended with $20 \mu / 3 \times$ SDS Laemmli sample buffer heated to $95-100^{\circ} \mathrm{C}$ for $5 \mathrm{~min}$. Immunoprecipitated proteins were separated into $15 \%$ SDS-PAGE gel, transferred onto PVDF membrane and detected using anti-EFEMP1 antibody (Abcam, Cambridge, UK) (see above).

\section{EGF-EFEMP1 competition experiment}

L3.6pl cells grown to $70 \%$ of apparent confluence were treated with $50 \mathrm{ng} / \mathrm{ml}$ recombinant human EGF (R\&D Systems) for 10 min at $37^{\circ} \mathrm{C}$ or with the irrelevant protein BSA (albumin Fraction $V$ for molecular biology, Carl Roth) in parallel under the same concentration and conditions. Cells were then subjected to coimmunoprecipitation (see above) using anti-EGFR antibody. Immunoprecipitation was analyzed by Western blotting using anti-EFEMP1 antibody (Abcam). Loading was controlled by Western blotting using anti-EGFR antibody (Cell Signaling, Danvers, MA, USA).

\section{Detection of EFEMP1-induced EGFR phosphorylation}

L3.6pl cells grown to $70 \%$ of apparent confluence were treated with $50 \mathrm{ng} / \mathrm{ml}$ purified recombinant human EFEMP1 (Abnova, Taipei City, Taiwan) for $48 \mathrm{~h}$ at $37^{\circ} \mathrm{C}$, or with the irrelevant protein BSA (albumin Fraction V for molecular biology, Carl Roth) in parallel under the same concentration and conditions. Cell lysates were prepared using Complete Lysis-M buffer (Roche) supple- mented with sodium vanadate. The amount of lysate was normalized to the protein concentration assayed by a BCA Protein Assay Kit (Pierce). Cell lysates were then subjected to Western blotting using a Phospho-EGF Receptor Antibody Sampler Kit (Cell Signaling) according to the manufacturer's instruction manual. EFEMP1-dependent phosphorylation of EGFR at the positions Tyr-845, Tyr-992, Tyr-1045, and Tyr-1068 was assayed. Antibody identifying total EGFR (Cell Signaling) was used as the loading control.

\section{FACE $^{\mathrm{TM}}$ EGFR: in-cell Western analysis for Tyr-992-phospho EGF}

An assay was performed using a FACE ${ }^{\mathrm{TM}}$ EGFR Y992 kit (Active Motif, Carlsbad, CA, USA) according to the manufacturer's instruction manual. A total of $10000 \mathrm{FG}$ or L3.6pl cells per well were seeded on 96-well plates, starved for $24 \mathrm{~h}$ and then activated for $10 \mathrm{~min}$ at $37^{\circ} \mathrm{C}$ with $\mathrm{FCS}$ or $40 \mathrm{ng} / \mathrm{ml}$ human recombinant EGF (R\&D Systems), or were left untreated. Cells were fixed by formaldehyde treatment and concentration of total EGFR or its phosphorylated form was determined via conventional ELISA. Wells containing the cells untreated with the first antibody were used as a blank. Four wells were assayed for each cell/treatment combination. Cell number was quantified colorimetrically following staining with crystal violet. These data were used for normalization of EGFR values to the cell number. Statistical significance was analyzed using Student's $t$-test.

\section{Phosphorylation of the proteins downstream to EGFR}

Effect of EFEMP1 treatment on activation of Akt- and MAPKsignal transduction pathways was investigated via detection of Akt phosphorylation in the position Thr-308 and Erk1, Erk2 phosphorylation in positions Tyr-202 and Tyr-204. The cell extracts prepared for studying EFEMP1-induced phosphorylation of EGFR (see above) were also used for investigation of the downstream proteins. Anti-phospho-Akt (Thr-308) antibody and anti-phospho-p44/42 MAPK (Thr-202/Tyr-204) (Cell Signaling) identifying Akt or Erk1 and Erk2, respectively, were used for Western blotting (see above).

\section{Inhibition of EGFR activity via treatment with PD15035}

A fraction of the same cell culture used for phosphorylation studies was treated with $50 \mathrm{ng} / \mathrm{ml}$ purified recombinant human EFEMP1 (Abnova) and with or without $2 \mu \mathrm{mol} / \mathrm{ml} \mathrm{PD153035}$ (Tocris, Bristol, UK) (Xu and Shu, 2007). Cell extracts were prepared (see above), normalized for protein concentration and subjected to Western blotting using anti-phospho-Akt (Thr-308) antibody and anti-phospho-p44/42 MAPK (Thr-202/Tyr-204).

\section{Acknowledgments}

We thank Dr. Brian W. Ziegelaar for critical reading of the manuscript and Martin Luckner for excellent technical assistance. This work was supported by grant KFO128/3 from the Deutsche Forschungsgemeinschaft.

\section{References}

Albig, A.R., Neil, J.R., and Schiemann, W.P. (2006). Fibulins 3 and 5 antagonize tumor angiogenesis in vivo. Cancer Res. $66,2621-2629$. 
Bruns, C.J., Harbison, M.T., Kuniyasu, H., Eue, I., and Fidler, I.J. (1999). In vivo selection and characterization of metastatic variants from human pancreatic adenocarcinoma by using orthotopic implantation in nude mice. Neoplasia 1, 50-62.

Bruns, C.J., Harbison, M.T., Davis, D.W., Portera, C.A., Tsan, R., McConkey, D.J., Evans, D.B., Abbruzzese, J.L., Hicklin, D.J., and Radinsky, R. (2000). Epidermal growth factor receptor blockade with $\mathrm{C} 225$ plus gemcitabine results in regression of human pancreatic carcinoma growing orthotopically in nude mice by antiangiogenic mechanisms. Clin. Cancer Res. 6, 1936-1948.

Chu, T.J. and Peters, D.G. (2008). Serial analysis of the vascular endothelial transcriptome under static and shear stress conditions. Physiol. Genomics 34, 185-192.

Eckstein, N., Servan, K., Girard, L., Cai, D., von Jonquieres, G., Jaehde, U., Kassack, M.U., Gazdar, A.F., Minna, J.D., and Royer, H.D. (2008). Epidermal growth factor receptor pathway analysis identifies amphiregulin as a key factor for cisplatin resistance of human breast cancer cells. J. Biol. Chem. 283, 739-750.

Fry, D.W., Kraker, A.J., McMichael, A., Ambroso, L.A., Nelson, J.M., Leopold, W.R., Connors, R.W., and Bridges, A.J. (1994). A specific inhibitor of the epidermal growth factor receptor tyrosine kinase. Science 265, 1093-1095.

Fu, L., Garland, D., Yang, Z., Shukla, D., Rajendran, A., Pearson, E., Stone, E.M., Zhang, K., and Pierce, E.A. (2007). The R345W mutation in EFEMP1 is pathogenic and causes AMDlike deposits in mice. Hum. Mol. Genet. 16, 2411-2422.

Giehl, K., Skripczynski, B., Mansard, A., Menke, A., and Gierschik, P. (2000). Growth factor-dependent activation of the Ras-Raf-MEK-MAPK pathway in the human pancreatic carcinoma cell line PANC-1 carrying activated K-ras: implications for cell proliferation and cell migration. Oncogene 19, 2930-2942.

Hashimoto, K., Farrow, B.J., and Evers, B.M. (2004). Activation and role of MAP kinases in 15d-PGJ2-induced apoptosis in the human pancreatic cancer cell line MIA PaCa-2. Pancreas 28, 153-159.

Kobayashi, N., Kostka, G., Garbe, J.H., Keene, D.R., Bachinger, H.P., Hanisch, F.G., Markova, D., Tsuda, T., Timpl, R., Chu, M.L., et al. (2007). A comparative analysis of the fibulin protein family. Biochemical characterization, binding interactions, and tissue localization. J. Biol. Chem. 282, 1180511816.

Koide, H., Muto, Y., Kasai, H., Kohri, K., Hoshi, K., Takahashi, S., Tsukumo, K., Sasaki, T., Oka, T., Miyake, T., et al. (1992). A site-directed mutagenesis study on the role of isoleucine23 of human epidermal growth factor in the receptor binding. Biochim. Biophys. Acta 1120, 257-261.

Koland, J.G., O'Brien, K.M., and Cerione, R.A. (1990). Expression of epidermal growth factor receptor sequences as $E$. coli fusion proteins: applications in the study of tyrosine kinase function. Biochem. Biophys. Res. Commun. 166, 90-100.

Marmorstein, L.Y., Munier, F.L., Arsenijevic, Y., Schorderet, D.F., McLaughlin, P.J., Chung, D., Traboulsi, E., and Marmorstein, A.D. (2002). Aberrant accumulation of EFEMP1 underlies drusen formation in Malattia Leventinese and age-related macular degeneration. Proc. Natl. Acad. Sci. USA 99, 1306713072.

Marmorstein, L.Y., McLaughlin, P.J., Peachey, N.S., Sasaki, T., and Marmorstein, A.D. (2007). Formation and progression of sub-retinal pigment epithelium deposits in Efemp1 mutation knock-in mice: a model for the early pathogenic course of macular degeneration. Hum. Mol. Genet. 16, 2423-2432.

McLaughlin, P.J., Bakall, B., Choi, J., Liu, Z., Sasaki, T., Davis, E.C., Marmorstein, A.D., and Marmorstein, L.Y. (2007). Lack of fibulin-3 causes early aging and herniation, but not macular degeneration in mice. Hum. Mol. Genet. 16, 3059-3070.

Miyamoto, S., Yagi, H., Yotsumoto, F., Horiuchi, S., Yoshizato, T., Kawarabayashi, T., Kuroki, M., and Mekada, E. (2007). New approach to cancer therapy: heparin binding-epidermal growth factor-like growth factor as a novel targeting molecule. Anticancer Res. 27, 3713-3721.

Nakamura, T., Fidler, I.J., and Coombes, K.R. (2007). Gene expression profile of metastatic human pancreatic cancer cells depends on the organ microenvironment. Cancer Res. 67, 139-148.

Ogiso, H., Ishitani, R., Nureki, O., Fukai, S., Yamanaka, M., Kim, J.H., Saito, K., Sakamoto, A., Inoue, M., Shirouzu, M., et al. (2002). Crystal structure of the complex of human epidermal growth factor and receptor extracellular domains. Cell 110, 775-787.

Parikh, A.A., Liu, W.B., Fan, F., Stoeltzing, O., Reinmuth, N., Bruns, C.J., Bucana, C.D., Evans, D.B., and Ellis, L.M. (2003). Expression and regulation of the novel vascular endothelial growth factor receptor neuropilin-1 by epidermal growth factor in human pancreatic carcinoma. Cancer 98, 720-729.

Rojas, M., Yao, S., and Lin, Y.Z. (1996). Controlling epidermal growth factor (EGF)-stimulated Ras activation in intact cells by a cell-permeable peptide mimicking phosphorylated EGF receptor. J. Biol. Chem. 271, 27456-27461.

Roybal, C.N., Marmorstein, L.Y., Vander Jagt, D.L., and Abcouwer, S.F. (2005). Aberrant accumulation of fibulin-3 in the endoplasmic reticulum leads to activation of the unfolded protein response and VEGF expression. Invest. Ophthalmol. Vis. Sci. 46, 3973-3979.

Schmitz, K.J., Wohlschlaeger, J., Lang, H., Sotiropoulos, G.C., Malago, M., Steveling, K., Reis, H., Cicinnati, V.R., Schmid, K.W., and Baba, H.A. (2008). Activation of the ERK and AKT signalling pathway predicts poor prognosis in hepatocellular carcinoma and ERK activation in cancer tissue is associated with hepatitis C virus infection. J. Hepatol. 48, 83-90.

Schonleben, F., Qiu, W., Bruckman, K.C., Ciau, N.T., Li, X., Lauerman, M.H., Frucht, H., Chabot, J.A., Allendorf, J.D., Remotti, H.E., et al. (2007). BRAF and KRAS gene mutations in intraductal papillary mucinous neoplasm/carcinoma (IPMN/IPMC) of the pancreas. Cancer Lett. 249, 242-248.

Seeliger, H., Camaj, P., Ischenko, I., Kleespies, A., De Toni, E.N., Thieme, S.E., Blum, H., Assmann, G., Jauch, K.W., and Bruns, C.J. (2009). EFEMP1 expression promotes in vivo tumor growth in human pancreatic adenocarcinoma. Mol. Cancer Res. 7, 189-198.

Semba, S., Moriya, T., Kimura, W., and Yamakawa, M. (2003). Phosphorylated Akt/PKB controls cell growth and apoptosis in intraductal papillary-mucinous tumor and invasive ductal adenocarcinoma of the pancreas. Pancreas 26, 250-257.

Shah, S.A., Potter, M.W., Hedeshian, M.H., Kim, R.D., Chari, R.S., and Callery, M.P. (2001). PI-3' kinase and NF-кB crosssignaling in human pancreatic cancer cells. J. Gastrointest. Surg. 5, 603-612.

Summy, J.M., Trevino, J.G., Baker, C.H., and Gallick, G.E. (2005). c-Src regulates constitutive and EGF-mediated VEGF expression in pancreatic tumor cells through activation of phosphatidyl inositol-3 kinase and p38 MAPK. Pancreas 31, 263-274.

Takeda, A., Osaki, M., Adachi, K., Honjo, S., and Ito, H. (2004). Role of the phosphatidylinositol 3'-kinase-Akt signal pathway in the proliferation of human pancreatic ductal carcinoma cell lines. Pancreas 28, 353-358.

Thalmeier, A., Dickmann, M., Giegling, I., Schneider, B., Hartmann, M., Maurer, K., Schnabel, A., Kauert, G., Moller, H.J., and Rujescu, D. (2008). Gene expression profiling of postmortem orbitofrontal cortex in violent suicide victims. Int. J. Neuropsychopharmacol. 11, 217-228.

Walker, F., Kato, A., Gonez, L.J., Hibbs, M.L., Pouliot, N., Levitz$\mathrm{ki}$, A., and Burgess, A.W. (1998). Activation of the Ras/mitogen-activated protein kinase pathway by kinase-defective epidermal growth factor receptors results in cell survival but not proliferation. Mol. Cell. Biol. 18, 7192-7204.

Weedon, M.N., Lango, H., Lindgren, C.M., Wallace, C., Evans, D.M., Mangino, M., Freathy, R.M., Perry, J.R., Stevens, S., Hall, A.S., et al. (2008). Genome-wide association analysis 
identifies 20 loci that influence adult height. Nat. Genet. 40, 575-583.

Xu, K. and Shu, H.K. (2007). EGFR activation results in enhanced cyclooxygenase-2 expression through p38 mitogen-activated protein kinase-dependent activation of the Sp1/Sp3 transcription factors in human gliomas. Cancer Res. 67, 6121-6129.

Yao, Z., Okabayashi, Y., Yutsudo, Y., Kitamura, T., Ogawa, W., and Kasuga, M. (2002). Role of Akt in growth and survival of PANC-1 pancreatic cancer cells. Pancreas 24, 42-46.

Yin, J. and Yu, F.S. (2009). ERK1/2 mediate wounding- and Gprotein-coupled receptor ligands-induced epidermal growth factor receptor activation via regulating ADAM17 and hepa- rin-binding EGF-like growth factor shedding. Invest. Ophthalmol. Vis. Sci. 50, 132-139.

Yip, W.K., Leong, V.C., Abdullah, M.A., Yusoff, S., and Seow, H.F. (2008). Overexpression of phospho-Akt correlates with phosphorylation of EGF receptor, FKHR and BAD in nasopharyngeal carcinoma. Oncol. Rep. 19, 319-328.

Zhang, B., Spandau, D.F., and Roman, A. (2002). E5 protein of human papillomavirus type 16 protects human foreskin keratinocytes from UV B-irradiation-induced apoptosis. J. Virol. $76,220-231$.

Received May 8, 2009; accepted August 8, 2009 